\title{
Bone Mineral Density: Clinical Relevance and Quantitative Assessment
}

\author{
Katherine N. Haseltine ${ }^{1}$, Tariq Chukir ${ }^{1}$, Pinar J. Smith ${ }^{2}$, Justin T. Jacob ${ }^{3}$, John P. Bilezikian ${ }^{4}$, and Azeez Farooki ${ }^{1}$ \\ ${ }^{1}$ Endocrinology Service, Department of Medicine, Memorial Sloan Kettering Cancer Center, New York, New York; ${ }^{2}$ Division of \\ Endocrinology, Diabetes, and Bone Disease, Icahn School of Medicine at Mount Sinai, New York, New York; ${ }^{3}$ Molecular Imaging and \\ Therapy Service, Department of Radiology, Memorial Sloan Kettering Cancer Center, New York, New York; and ${ }^{4}$ Division of \\ Endocrinology, Vagelos College of Physicians and Surgeons, Columbia University, New York, New York
}

\begin{abstract}
Learning Objectives: On successful completion of this activity, participants should be able to describe (1) the benefits and limitations of evaluating bone mineral density by dual-energy x-ray absorptiometry and FRAX to assess fracture risk; (2) the uses of various pharmacologic therapies available for
\end{abstract} the treatment of osteoporosis; and (3) the process of monitoring and ensuring patient response to osteoporosis therapy.

Financial Disclosure: Dr. Farooki has received consulting fees from Amgen and has been an Amgen advisory board member. Dr. Bilezikian has received consulting fees from Radius and Amgen. This work was supported in part by NIH/NCI Memorial Sloan Kettering Cancer Center support grant P30 CA008748. The authors of this article have indicated no other relevant relationships that could be perceived as a real or apparent conflict of interest.

CME Credit: SNMMI is accredited by the Accreditation Council for Continuing Medical Education (ACCME) to sponsor continuing education for physicians. SNMMI designates each JNM continuing education article for a maximum of 2.0 AMA PRA Category 1 Credits. Physicians should claim only credit commensurate with the extent of their participation in the activity. For CE credit, SAM, and other credit types, participants can access this activity through the SNMMI website (http://www.snmmilearningcenter.org) through April 2024.

Bone mineral density (BMD) measurement by dual-energy $\mathrm{x}$-ray absorptiometry (DXA) is an internationally accepted standard-of-care screening tool used to assess fragility-fracture risk. Society guidelines have recommended which populations may benefit from DXA screening and the use of the fracture risk assessment tool (FRAX) to guide decisions regarding pharmacologic treatment for osteoporosis. According to the U.S. National Osteoporosis Foundation guidelines, postmenopausal women and men at least 50 y old with osteopenic BMD warrant pharmacologic treatment if they have a FRAX-calculated 10 -y probability of at least $3 \%$ for hip fracture or at least $20 \%$ for major osteoporotic fracture. Patients with osteoporosis defined by a clinical event, namely a fragility fracture, or with an osteoporotic BMD should also be treated. Patients who are treated for osteoporosis should be monitored regularly to track expected gains in BMD by serial DXA scans. With some drug therapies, BMD targets can be reached whereby further improvements in BMD are not associated with further reductions in fracture risk. Although reaching this target might suggest a stopping point for therapy, the reversibility of most treatments for osteoporosis, except for the bisphosphonates, has dampened enthusiasm for this approach. In the case of denosumab, it is now apparent that stopping therapy at any point can lead to an increase in multiplefracture risk. For patients who do not respond to antiosteoporosis pharmacologic therapy with an improvement in BMD, or who have an incident fragility fracture on therapy, secondary causes of osteoporosis or non-compliance with medical therapy should be considered.

Key Words: bone mineral density; DXA; fracture risk; osteoporosis; FRAX

J Nucl Med 2021; 62:446-454

DOI: 10.2967/jnumed.120.256180

Received May 14, 2020; revision accepted Sep. 23, 2020.

For correspondence or reprints contact: Azeez Farooki, Memorial Sloan Kettering Cancer Center, 1275 York Ave., New York, NY 10065.

E-mail: farookia@mskcc.org

Published online Dec. 11, 2020.

COPYRIGHT (C 2021 by the Society of Nuclear Medicine and Molecular Imaging.
$\mathbf{O}$ steoporosis is highly prevalent but underdiagnosed and undertreated, partly because it is often clinically undetected until a fragility fracture occurs. Among adults aged greater than 50 y, 1 of 3 women and 1 of 5 men will experience a fragility fracture (1). Hip fractures are associated with more than a $20 \%$ mortality rate at $1 \mathrm{y}$ (2), and about $50 \%$ of patients lose their ability to live independently (3). Spine fractures are similarly painful, impair quality of life, and, often, with the development of height loss and kyphosis, contribute to a loss of self-esteem (4). Figure 1 shows common fractures in the United States in 2005 (5).

The components of bone are both inorganic (hydroxyapatite crystals composed of calcium and phosphate) and organic (90\% type I collagen plus other noncollagenous proteins). Lamellar type I collagen is strengthened by pyridinoline crosslinks between the collagen fibrils. The concerted and coordinated activities of osteoclasts (bone-resorbing cells) and osteoblasts (bone-forming cells) constantly remodels bone, a dynamic tissue. The ratio of the internal surface area to the mineralized bone matrix volume determines the accessibility of remodeling the mineralized bone matrix and its vulnerability to deterioration when remodeling becomes unbalanced (6). The surface area-to-mineralized bone ratio is low in cortical bone and high in trabecular bone (6). Sites that have high trabecular bone content (posterior-anterior spine) are more metabolically active; therefore, a significant change in bone mineral density (BMD) is likely to occur earlier at the spine than at the hip or forearm (7).

Osteoporosis is associated with an imbalance in bone remodeling, in which there is relatively greater bone resorption than bone formation. However, the actual rate of bone resorption or bone formation could be above normal (accelerated bone remodeling), normal, or below normal (reduced bone remodeling). In each case, the result of the bone remodeling process leads to a net loss of bone material because of an imbalance in the process, independent of the rate of bone remodeling. Deterioration of skeletal microstructure and bone strength, both associated with loss of bone material, leads to increased susceptibility to fracture (Fig. 2) (8). 


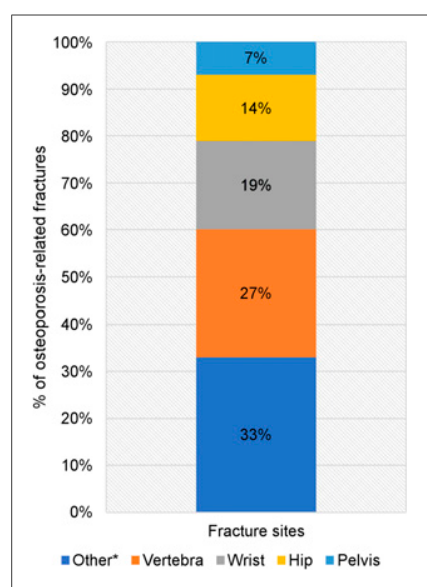

FIGURE 1. Sites of fractures caused by osteoporosis in United States in 2005. There were $\sim 2$ million osteoporosis-related fractures in 2005 in United States: $29 \%$ in men, $14 \%$ in nonwhite Americans, and $73 \%$ at nonvertebral sites. *Radius, humerus, clavicle, hands/fingers, patella, and tibia/fibula (Adapted with permission (5).)

macotherapy used to lower the osteoporotic fracture risk in patients.

\section{DXA: THE GOLD STANDARD FOR ASSESSING FRACTURE RISK}

Current DXA systems have similar operating principles, comprising a radiation source emitting 2 x-ray energies, a radiation detector, and a table to support the patient. Images and quantitative measurement of bone and soft-tissue density are produced by software that assesses the difference in attenuation between the 2 different energies (12). Before the advent of current DXA systems, the earliest attempts to measure BMD comprised plain radiographs for quantitative and qualitative morphometry (13). Nuclear medicine would play a role in early BMD measurement with singlephoton absorptiometry and dual-photon absorptiometry. Although current systems use an x-ray tube as the radiation source, the earliest forms of photon absorptiometry used radionuclides as the photonemitting source. ${ }^{125} \mathrm{I}(27.3 \mathrm{keV})$ was most commonly used to

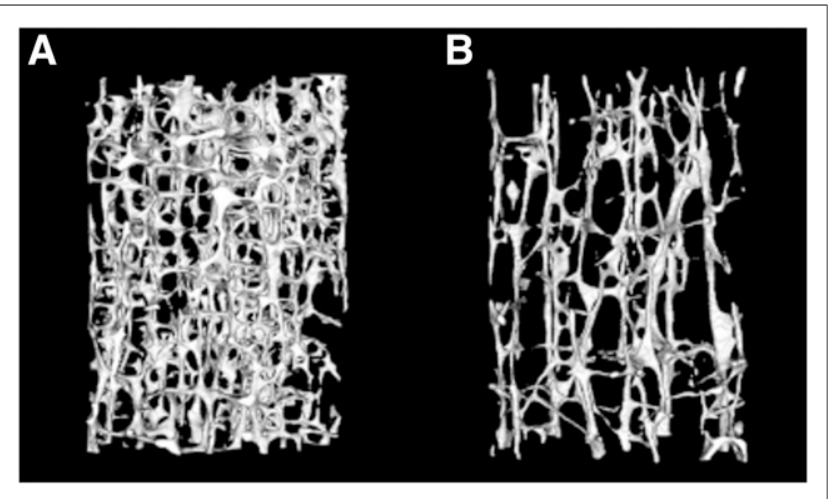

FIGURE 2. Three-dimensional micro-CT. Loss of horizontal trabeculae in osteoporosis is seen in 52-y-old woman (A) and 84-y-old woman (B) with vertebral fracture. (Reprinted with permission of (8).) generate the single photon beam, whereas dual-photon absorptiometry used ${ }^{153} \mathrm{Gd}$ with its 2 distinct photoelectric peaks (44/100 keV). These radionuclide-based techniques have led to current DXA systems using $\mathrm{x}$-ray photons.

DXA is a 2-dimensional measurement of bone mass, or a measurement of area. Since bone depth is not a factor, bone size can affect apparent BMD. Essentially, 2 vertebrae with identical volumetric densities can have different areal densities based on size (13). As a result, the larger the bone the higher the apparent BMD. Although DXA is still considered the gold standard for assessing fracture risk, other modalities can offer potential value in the assessment of bone mass. Quantitative CT is a volumetric measurement of bone density. Quantitative CT of the spine spatially isolates trabecular bone, which is metabolically more active than cortical bone, allowing changes in bone mass to be observed at a greater rate than is possible with DXA (13). Although quantitative CT and DXA have the same ability to predict vertebral fractures in postmenopausal women, there is lack of evidence to support this position for men (14). For both men and women, the International Society for Clinical Densitometry (ISCD) does not recommend spine quantitative CT for the prediction of hip fractures.

Recent data suggest that modern nuclear medicine departments can assess bone mass. In a study by Huang et al., quantitative bone SPECT/CT with ${ }^{99 m}$ Tc-MDP was found to be a viable tool for clinical quantification of bone metabolism in patients with osteoporosis (15). On the other hand, using the CT component of SPECT/CT and PET/CT can offer the nuclear medicine physician the opportunity to analyze CT Hounsfield units, which can provide useful information on bone mass and the need for further imaging.

DXA has an extremely low radiation dose (1-10 $\mu \mathrm{Sv})$, comparable to natural background radiation received each day $(7 \mu \mathrm{Sv})$ (16). Full-table DXA systems can obtain BMD measurements at several sites (e.g., lumbar spine, hip, forearm), whereas peripheral DXA systems measure only the peripheral skeleton such as the forearm. Full-table systems are used widely in clinical practice and research for osteoporosis assessment, with the lumbar spine and hip serving as principal sites for diagnosis and therapeutic decision making (12). Vertebral fractures can be identified with good sensitivity and specificity by DXA. This adaptation, known as vertebral fracture assessment, uses a software program and evaluation by the semiquantitative method of Genant et al. (Fig. 3) $(12,17,18)$.

The standard BMD measurement is by T-score, which is a comparison between the patient's BMD and the mean BMD of a healthy young adult. The T-score unitage is most simply described as the number of SDs that the patient's score is below the control value. Osteoporosis is defined by a T-score of no more than -2.5 (19). Like vertebral fracture assessment, DXA has also been adapted to semiquantitatively assess skeletal microstructure. Using a software program that determines the extent to which the lumbar vertebra is homogeneous or heterogeneous, a noninvasive assessment of skeletal microstructure is possible. This adaptation, known as the trabecular bone score (TBS), was approved by the FDA in 2012 (20). TBS, an independent predictor of fracture risk, adds to information obtained by the T-score and the fracture risk assessment tool (FRAX; Centre for Metabolic Bone Diseases, University of Sheffield). The Medimaps Group, which produced the TBS iNsight (Osteo) software, proposes the following interpretation of TBS values. For postmenopausal women, a TBS of at least 1.350 is normal; $1.2-1.350$ indicates partially degraded bone; and 1.2 or less indicates the lowest tertile of skeletal microstructure (21). TBS is not used alone for treatment 


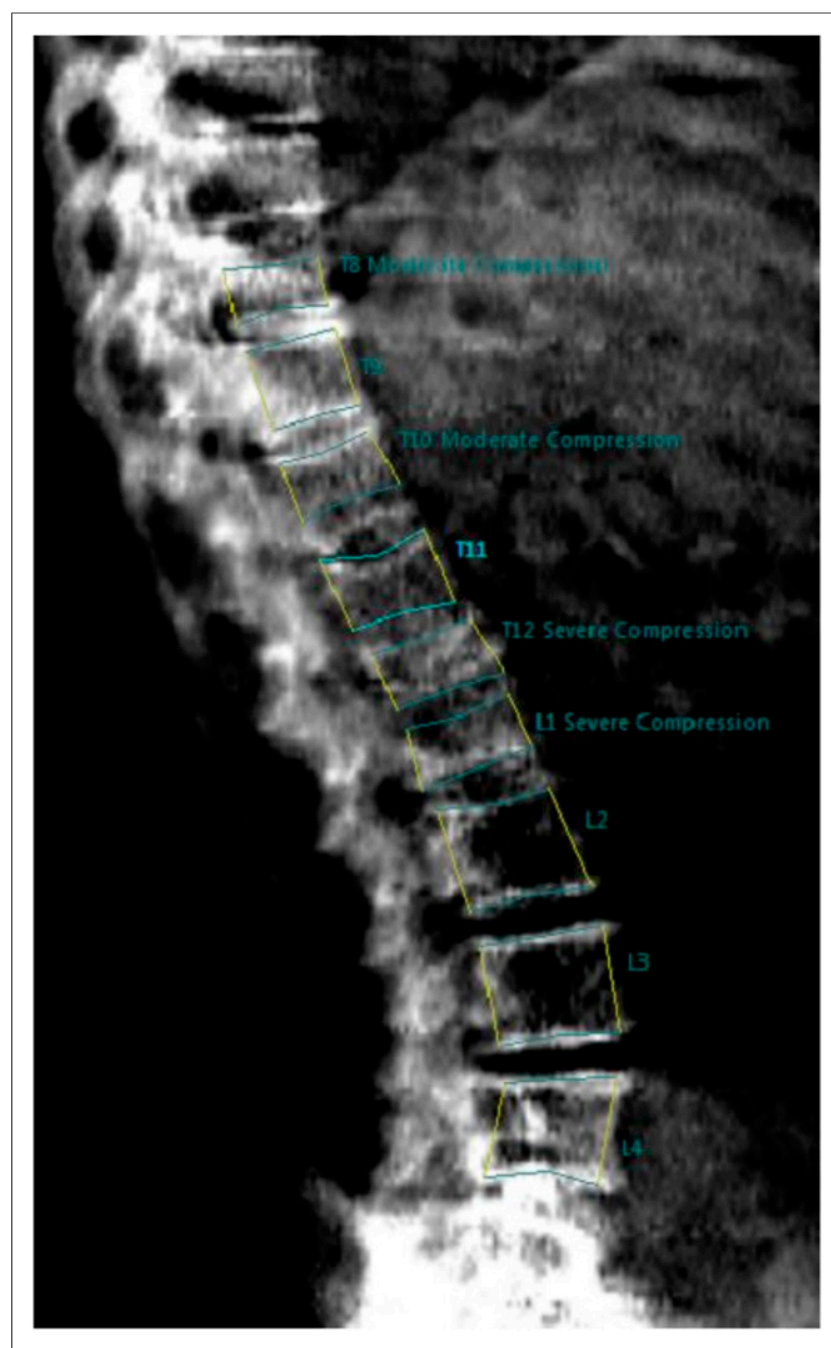

FIGURE 3. Vertebral fracture assessment depicting multiple compression deformities.

decision making, but it is helpful as an adjunct to the 2 modalities that are used for clinical decision making, namely BMD and FRAX. Guidelines on the use of TBS in clinical practice have been published by the ISCD $(22,23)$. The semiquantitative assessment is now incorporated into the FRAX software (24), adding to its predictive power. ISCD suggests using the TBS-adjusted World Health Organization FRAX in treatment decision making (22), as described in the "Evaluating Fracture Risk Using FRAX" section. An advantage of TBS is that it is not affected by osteophytes or other ectopic skeletal calcifications, unlike DXA $(25,26)$. Nonetheless, abdominal obesity can potentially introduce an artifact in the TBS measurement $(27,28)$.

\section{Recommendations for DXA Screening}

DXA screening for osteoporosis is important because osteoporosis is asymptomatic until a fracture occurs. The National Osteoporosis Foundation, American Society for Bone and Mineral Research, and ISCD recommend BMD testing by DXA for women aged at least $65 \mathrm{y}$ and men aged at least $70 \mathrm{y}$, as well as, according to risk factor profile, postmenopausal women and men aged at least 50 y (29). Risk factors for fragility fractures include advancing age, low body weight, excessive alcohol consumption, current smoking, family history of osteoporosis, early menopause, hyperthyroidism, hyperparathyroidism, hypogonadism, history of organ transplantation, malabsorption, and treatment with certain medications, such as long-term glucocorticoids, aromatase inhibitors, or androgen deprivation therapies $(5,30)$.

\section{DXA SCANNING IN SPECIAL POPULATIONS}

\section{Patients Treated with Chronic Glucocorticoid Therapy}

The 2017 American College of Rheumatology guidelines on glucocorticoid-induced osteoporosis prevention and treatment recommend BMD testing for fracture risk assessment in all adults receiving chronic glucocorticoid therapy ( $\geq 3 \mathrm{mo}$ ) aged at least $40 \mathrm{y}$ or those younger than $40 \mathrm{y}$ whose fracture risk is high because of a previous fracture or other significant osteoporotic risk factors (31).

\section{Recipients of Solid-Organ Transplants}

Recipients of solid-organ transplants experience rapid bone loss in the first 6-12 mo after transplantation (32), a time when fractures are most common $(33,34)$. There is no consensus on prevention of bone loss and fractures through medical management in patients undergoing solid-organ transplantation. The American College of Rheumatology recommends treatment, following the same age guidelines mentioned above for glucocorticoid-induced osteoporosis prevention and treatment in this population (31).

\section{Women with Premature Menopause}

Premature menopause is defined as menopause in women younger than $40 \mathrm{y}$. Etiologies of premature menopause include chemotherapy, oophorectomy, or medical conditions (e.g., autoimmune diseases). Women who experience premature menopause after chemotherapy have more rapid bone loss than their counterparts who maintain menses (35), suggesting that it is reasonable to screen for osteoporosis and fragility fractures by DXA in this population.

\section{Men with Hypogonadism}

Men with primary hypogonadism, secondary hypogonadism, or androgen insensitivity have increased bone turnover and decreased bone density. Significant loss of BMD can occur in as little as 6 mo, as demonstrated in men starting androgen deprivation therapy for prostate cancer. Men who are found to be hypogonadal should have a baseline DXA measurement, as well as intermittent follow-up (36).

\section{Osteomalacia}

Osteomalacia, a condition distinct from osteoporosis in which large amounts of unmineralized bone (osteoid) are present, is most commonly caused by severe vitamin D deficiency. In these patients, DXA may show decreased BMD at the spine, hip, and forearm, generally indistinguishable from osteoporosis on DXA alone. A complete laboratory work-up, including calcium, phosphate, alkaline phosphatase, and vitamin D levels, is vital for the clinician to make the correct diagnosis (37). Successful treatment of this condition causes remineralization of the skeleton and often produces a dramatic increase in BMD.

\section{DXA INTERPRETATION AND POSITIONING}

DXA results may be confounded by several issues, including positioning errors, artifacts, vertebral fractures, vertebral osteoarthritis, scoliosis, and nonadherence to ISCD guidelines on serial DXA measurements (Figs. 4 and 5). These issues may adversely affect the calculation of BMD and should be considered when 


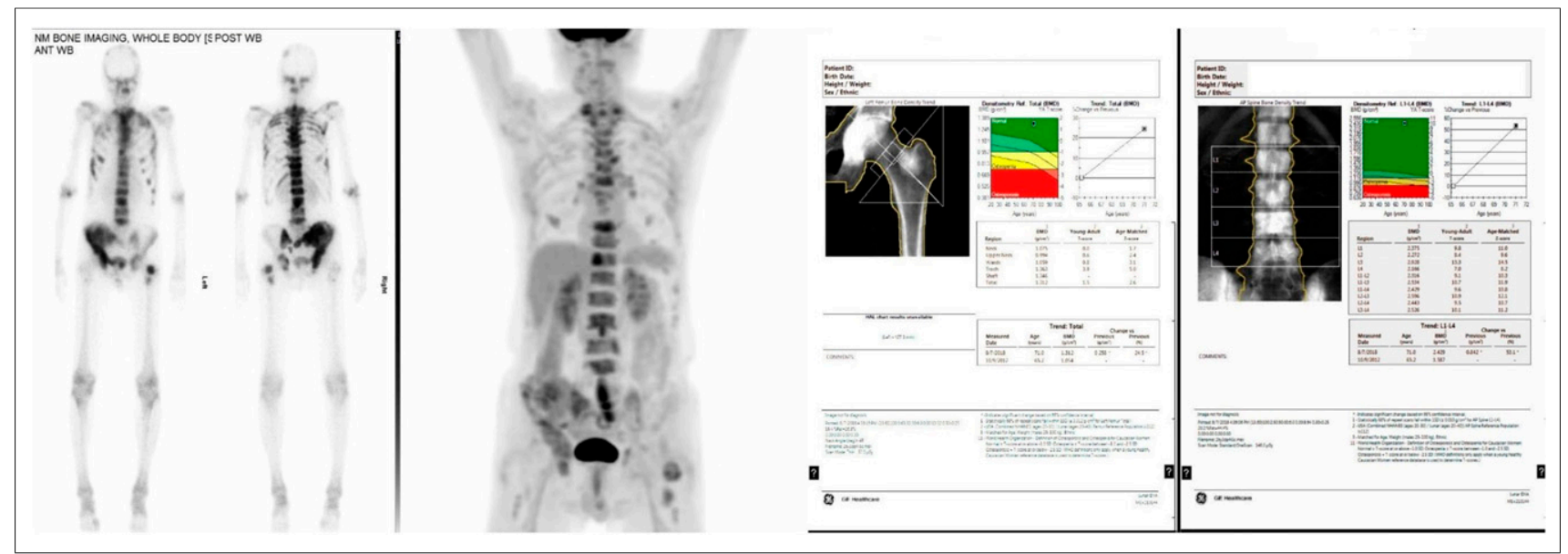

FIGURE 4. Osteoblastic metastases limiting assessment. ISCD recommended imaging techniques to include nondominant forearm to avoid inaccurate measurement.

interpreting DXA images. Suboptimal internal hip rotation is a common pitfall adversely affecting the BMD of the femoral neck and total hip. Continuous technologist training to ensure adherence to ISCD positioning guidelines is of the utmost importance. Another pitfall is the administration of oral contrast or radionuclides preceding the DXA scan. Failure to recognize the oral contrast medium overlying the lumbar spine could lead to reporting a falsely high BMD.

The effect of radionuclide administration before DXA studies has been debated for years. On GE Healthcare Lunar systems, the effect on BMD varies between patients and is dependent on the site scanned and dose administered (38). To avoid the radionuclide effect on BMD, DXA can be performed before the administration of the radiopharmaceutical. If this is not possible, it is recommended that the DXA study be postponed for $24-48 \mathrm{~h}$ after the nuclear medicine study (38).

Discrepancies among BMD measurements also can be observed when different DXA machines are used. Each manufacturer uses a different reference population database to calculate the T-score at several sites (39). The ISCD recommends calculating the least significant change (LSC) at each center performing DXA (30). For centers with more than 1 technologist, the LSC is measured

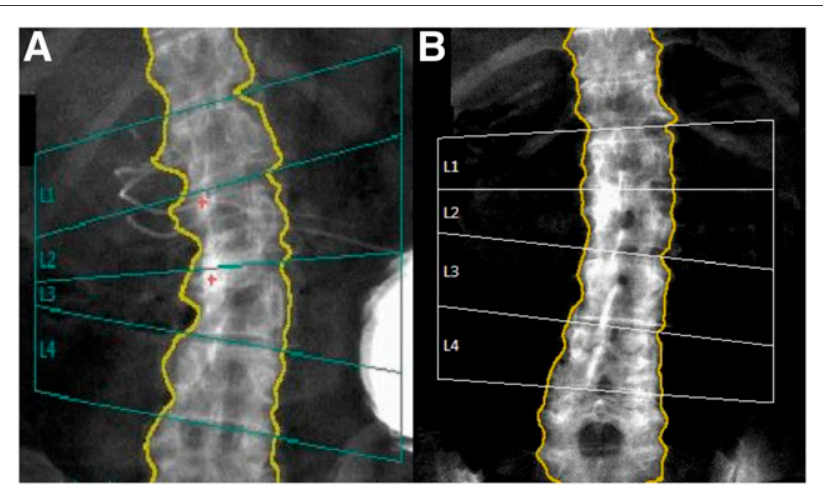

FIGURE 5. Cases of entities that confound DXA. (A) Scoliotic spine with hepatic arterial infusion pump limiting assessment. (B) Degenerative spine with multilevel sclerotic artifacts falsely increasing BMD. at each skeletal site by each technologist and is calculated by multiplying 2.77 times the precision error; it is best expressed as an absolute value $\left(\mathrm{g} / \mathrm{cm}^{2}\right)$. The provided DXA manufacturer-specific default precision error value should not be used in lieu of a center-specific calculated LSC.

Discrepancies among BMD measurements can also be observed between DXA machines of the same manufacturer. The ISCD currently provides instructions on cross-calibration of hardware or scanners across different centers. It is not currently possible to quantitatively compare BMD values or to calculate the LSC between centers without cross-calibration (14). Therefore, patients are encouraged to return to the same DXA scanner as was used for their prior measurement for proper serial BMD measurement.

A stable or increased BMD correlates with the antifracture efficacy of pharmacotherapy. When DXA follow-up testing shows BMD deterioration despite pharmacotherapy, collaboration between readers of the DXA scans and physicians treating osteoporosis is particularly important, because substantial loss of BMD (greater than the LSC) may require therapy adjustment or further assessment. Notably, substantial weight loss can lead to reduced BMD, despite effective therapy.

\section{INDICATIONS FOR TREATMENT BASED ON BMD}

Guidelines from the National Osteoporosis Foundation and other societies recommend that, after hip or vertebral fractures, patients should receive pharmacotherapy as described in the "Treatment Options" section. Both symptomatic and asymptomatic spine fractures (detected on vertebral imaging modalities, as shown through loss of vertebral height) warrant pharmacotherapy (Table 1). The Genant semiquantitative grading system is a useful method to evaluate subclinical osteoporotic vertebral fractures (18). These morphometric fractures are as predictive of future fractures as are clinically overt events $(40,41)$. Notably, both compression fractures of the spine and osteophyte formation may artificially elevate the calculated 2-dimensional BMD measurement on a DXA scan, resulting in false reassurance of normal BMD when in fact the patient may have osteoporosis. Typically, the experienced reader of DXA scan will rather quickly identify and exclude the artifact in a given vertebra from the analysis. The 
TABLE 1

Indications for Treatment to Reduce Osteoporosis or Osteopenia in Postmenopausal Women and Men Aged $\geq 50$ Years with Elevated Fracture Risk

\begin{tabular}{ll}
\hline \multicolumn{1}{c}{ Indication } & \multicolumn{1}{c}{ Description } \\
\hline $\begin{array}{l}\text { Hip or vertebral fracture } \\
\text { Vertebral fracture }\end{array}$ & $\begin{array}{l}\text { Clinically apparent hip or vertebral fracture } \\
\text { Vertebral fracture detected on imaging }\end{array}$ \\
$\begin{array}{ll}\text { T-score } \\
\leq-2.5\end{array}$ & $\begin{array}{l}\text { T-score of } \leq-2.5 \text { at femoral neck, total hip, or lumbar spine } \\
-1 \text { to }-2.5\end{array}$ \\
$\begin{array}{r}\text { T-score of }-1 \text { to }-2.5 \text { and } 10-y \text { probability of } \geq 3 \% \text { for hip fracture or of } \geq 20 \% \text { for } \\
\text { major osteoporotic fracture* estimated with FRAX }\end{array}$
\end{tabular}

${ }^{*}$ Hip, clinical spine, humerus, or wrist.

ISCD states that at least 2 evaluable vertebrae must be present for a lumbar spine measurement to be reportable.

There are 2 ways to establish the diagnosis of osteoporosis. The first is in patients with no history or evidence of a fragility fracture. In this population, osteoporosis can be diagnosed if the Tscore is no more than -2.5 (42). The second is the fragility-fracture event, which supersedes DXA measurement. In other words, BMD might be in the osteopenic range (T-score between -1.0 and $-2.5)$, but if a fragility fracture is present, the correct clinical diagnosis is osteoporosis. Although patients with osteopenia have a lower fracture risk than patients with osteoporosis, the incidence of osteopenia is much higher than that of osteoporosis; therefore, most patients with fractures do not have preexisting osteoporosis based on DXA measurement. Treatment is effective in both situations (43).

\section{Evaluating Fracture Risk Using FRAX}

Another indication for treatment relates to those who have not sustained a fragility fracture and whose T-score is in the osteopenic range. In this setting, the FRAX calculation can be helpful. If these patients meet treatment thresholds by FRAX, they are candidates for pharmacologic treatment (Table 1). The FRAX score is calculated using a computer-based algorithm that estimates the $10-y$ probability of both a major osteoporotic fracture (hip, clinical spine, humerus, or wrist) and a hip fracture (44). FRAX calculations integrate a patient's age, sex, race, weight, height, family medical history, tobacco use, glucocorticoid use, history of rheumatoid arthritis, evidence of secondary osteoporosis, and excessive alcohol use, in addition to femoral neck BMD. FRAX is a well-validated tool with over a million person-years of observation (45). The FRAX score takes into account the risk of death, because the greater the risk of death in a 10 -y period, the lower the risk of sustaining a fracture for the same risk factors.

The values of individual risk factors for an osteoporotic fracture and mortality data are country-specific. Thus, FRAX scores necessarily vary from country to country for individuals who may present with the same risk profile (24). FRAX-based indications for therapy depend on high risk as determined by countryspecific FRAX scores of at least $3 \%$ or at least $20 \%$ for hip or major osteoporotic fractures, respectively (Table 1) $(44,46)$.

Diabetes mellitus is known to confer an additional increased fracture risk but is not integrated as an associated clinical risk factor in the FRAX calculator (47). For a given T-score, diabetes mellitus is associated with greater risk. Although patients with diabetes mellitus do not have rheumatoid arthritis, many clinicians will adjust the FRAX score by selecting the box labeled "rheumatoid arthritis" (24) to account for the additional risk of having diabetes mellitus. Notably, there are other risk factors not considered by FRAX, such as risk of falls and rate of bone loss.

\section{TREATMENT OPTIONS}

\section{Antiresorptive Agents}

Antiresorptive agents reduce bone turnover, improve BMD, and reduce fracture risk. They represent the mainstay of therapy for osteoporosis. Antiresorptive agents (Table 2) inhibit osteoclast activity, which causes decreased bone resorption, and because bone turnover is a tightly coupled process, antiresorptive agents reduce osteoblast activity and bone formation. The generalized reduction in bone turnover is beneficial in 2 ways. General bone remodeling is reduced, and the balance between bone resorption and bone formation becomes positive because resorption is inhibited to a greater extent than formation.

Bisphosphonates bind to bone mineral with variable strength, and studies evaluating discontinuation suggest a persistent antifracture effect, at least in the short term. The FDA recommends bisphosphonate drug holidays after 3-5 y of therapy because of reported rare adverse effects with long-term bisphosphonate use (48) and evidence of persistent antifracture effects. Resumption of bisphosphonate therapy after a drug holiday may be indicated in

TABLE 2

Mechanism of Action with Antiresorptive and Anabolic Therapies

\begin{tabular}{ccc}
\hline \multicolumn{1}{c}{ Type of drug } & $\begin{array}{c}\text { Bone } \\
\text { formation }\end{array}$ & $\begin{array}{c}\text { Bone } \\
\text { resorption }\end{array}$ \\
\hline $\begin{array}{c}\text { Antiresorptive therapy } \\
\text { Anabolic therapy } \\
\text { (e.g., PTH analog) }\end{array}$ & $\begin{array}{c}\text { Decrease } \\
\text { Transient } \\
\text { increase }\end{array}$ & $\begin{array}{c}\text { Decrease } \\
\text { Eventual } \\
\text { increase }\end{array}$ \\
$\begin{array}{c}\text { Anabolic therapy } \\
\text { (e.g., sclerostin } \\
\text { antibody) }\end{array}$ & $\begin{array}{c}\text { Transient } \\
\text { increase }\end{array}$ & Decrease \\
\hline PTH $=$ parathyroid hormone. & & \\
\hline
\end{tabular}


patients who experience a fracture or show significant BMD loss (7). According to the American Society for Bone and Mineral Research 2015 guidelines, a drug holiday should be considered after 5 y for postmenopausal women treated with oral bisphosphonates and after $3 \mathrm{y}$ for those treated intravenously (49).

Denosumab, a monoclonal antibody that targets the boneresorbing ligand known as RANKL, is another common antiresorptive treatment. Unlike bisphosphonates, denosumab does not bind to bone mineral. It circulates as all antibodies do. Because of its specific affinity for RANKL, denosumab binds to and inactivates RANKL. Loss of RANKL leads to a profound reduction in osteoclast activity. The approved dose $(60 \mathrm{mg})$ by subcutaneous injection lasts for approximately $6 \mathrm{mo}$, at which time another dose is administered. The effects of denosumab are rapidly reversible. If a dose is missed, the antiresorptive effect dissipates. This rebound effect has been demonstrated in a study showing that BMD decreased to baseline values and bone turnover markers increased to values higher than baseline by 12 mo after the discontinuation of a 2-y treatment period with denosumab (7). Cases of multiple spine fractures have been observed in high-risk patients who discontinued denosumab. If denosumab is stopped, a bisphosphonate should be prescribed to prevent BMD loss (46). For denosumab, a drug holiday is not recommended.

Other antiresorptive agents include calcitonin, raloxifene, and hormone or estrogen replacement therapies. All of these treatments allow more time for secondary mineralization in the existing bone tissue mass, which increases BMD and the mechanical resistance of bone to loading (50).

Calcitonin, a weak antiresorptive agent, is no longer used to treat osteoporosis because of concerns of increased cancer risk and its weak therapeutic benefit $(51,52)$. Although maintaining the function of osteoblasts and osteocytes, bone remodeling is suppressed to the premenopausal range by estrogens and raloxifene (a selective estrogen receptor modulator that acts as an estrogen agonist in bone) (50). Raloxifene also has been shown to reduce the incidence of breast cancer (50). It has not been found to reduce the risk of hip fracture, however, possibly because of its relatively weaker antiresorptive potency. Randomized controlled trials of FDA-approved antiresorptive and anabolic therapies are compared in Table $3(53,54)$.

\section{Anabolic Agents}

In contrast to antiresorptive agents, anabolic agents promote bone formation and activate bone remodeling (Table 2). Generally, anabolic agents are given to men or postmenopausal women who have a very high risk of subsequent fractures, or severe osteoporosis (generally defined as either a T-score of $\leq-3.5$ in the absence of a fracture or a T-score of $\leq-2.5$ in the presence of a fragility fracture); patients with contraindications to oral bisphosphonates (e.g., esophageal emptying disorders or esophageal strictures); and patients for whom other therapies have failed (e.g., the patient sustained a fracture or had significant BMD loss while receiving other therapy).

Two human parathyroid hormone analogs, teriparatide and abaloparatide, are anabolic agents that produce a quick increase in bone-formation markers and, later, an increase in boneresorption markers; this interval is described as an anabolic window (Fig. 6) (55). These agents are contraindicated in patients with a history of external-beam radiation therapy because of a theoretic risk of osteosarcoma, and in patients with active bone metastases. However, with teriparatide now approved for almost 2 decades, no oncogenic signals have emerged. Both teriparatide and abaloparatide are administered subcutaneously as a daily dose for no more than $2 \mathrm{y}$.

A recently introduced anabolic agent and monoclonal antibody, romosozumab, binds the osteocyte-derived protein sclerostin,

TABLE 3

FDA-Approved Antiresorptive and Anabolic Therapies Used in Randomized Clinical Trials $(53,54)$

\begin{tabular}{|c|c|c|c|c|}
\hline Drug & Name of study & No. of patients & $\mathrm{RR} \%$ of vertebral Fx & $\mathrm{RR} \%$ of hip Fx \\
\hline \multicolumn{5}{|c|}{ Antiresorptive therapies } \\
\hline Calcitonin & PROOF & 1,255 & 36 & NS \\
\hline Raloxifene & MORE & 7,704 & $30-55$ & NS \\
\hline HRT/ERT & WHI & 16,608 & 34 & $34-39$ \\
\hline Alendronate & FIT-1 & 2,027 & 47 & 51 \\
\hline Risedronate & VERT & 2,458 & $41-49$ & NA \\
\hline Risedronate & HIP-OP & 5,445 & NA & 40 \\
\hline Ibandronate & BONE & 2,946 & 52 & NS \\
\hline Zoledronic acid & HORIZON & 7,765 & 70 & 41 \\
\hline Denosumab & FREEDOM & 7,868 & 68 & 40 \\
\hline \multicolumn{5}{|l|}{ Anabolic therapies } \\
\hline Teriparatide & NCT00670501* & 1,637 & 84 & NA \\
\hline Abaloparatide & ACTIVE & 2,463 & 86 & 43 (NV Fx) \\
\hline Romosozumab & FRAME & 7,180 & 83 & NS \\
\hline $\begin{array}{l}{ }^{\star} \text { ClinicalTrials.gov } \\
\mathrm{RR}=\text { relative risk } \\
\text { gnificant; } \mathrm{NA}=\text { not }\end{array}$ & fracture; HRT & normone replace & erapy/estrogen replac & erapy; NS = not \\
\hline
\end{tabular}




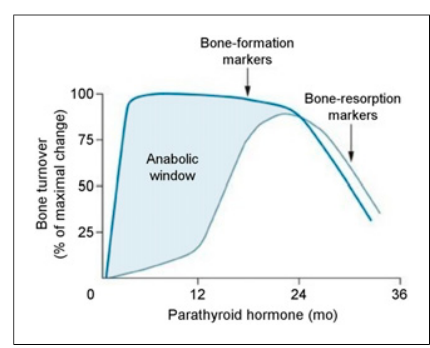

FIGURE 6. Anabolic window. With 2 anabolic agents that have mechanism of action similar to that of parathyroid hormone analogs, there are rapid increases in bone-formation markers followed by increases in bone-resorption markers. (Reprinted with permission of (55).)

sorption markers are below baseline.

One trial of romosozumab versus alendronate (no placebo control) noted an increased risk of myocardial infarction and stroke. In other trials of romosozumab with placebo controls, this imbalance was not seen (56-58). The FDA has issued a black box warning stating that romosozumab is contraindicated for patients with a history of myocardial infarction or stroke in the past year and should be discontinued for patients who develop a myocardial infarction or stroke on treatment.

\section{Risks of Treatments for Osteoporosis}

It is important to balance a discussion of benefits with risks, especially when discussing treatments for osteoporosis, because several classes have been associated with rare but serious side effects. Unfortunately, media reports have disproportionately magnified these risks and have not effectively communicated the benefits that most patients gain by taking these therapies. Figure 7 exemplifies this point by describing, for example, the benefits of bisphosphonates relative to the risks of its adverse effects and of other rare events, such as a motor vehicle accident or homicide (59). Largely because of fear of adverse effects, the percentage of patients who received an approved therapy for

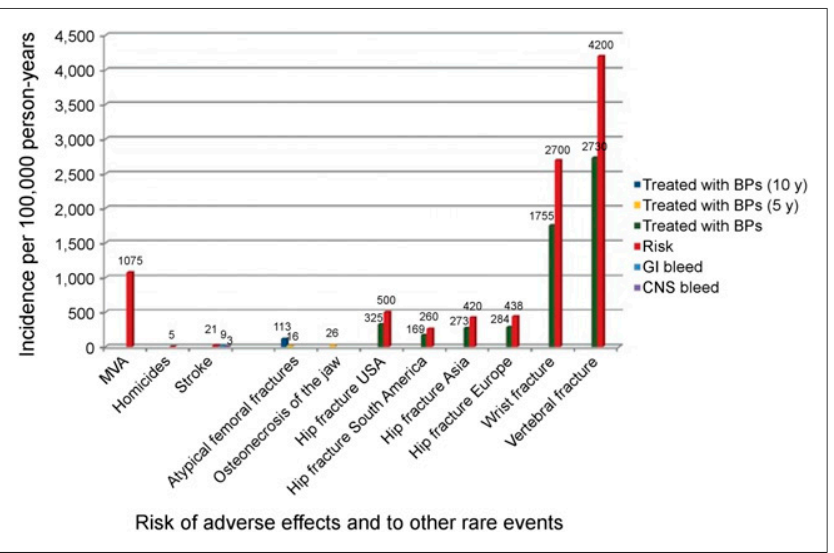

FIGURE 7. Risks associated with bisphosphonate use relative to both adverse effects and other rare events. BPs = bisphosphonates; CNS = central nervous system; $\mathrm{Gl}=$ gastrointestinal; $\mathrm{MVA}=$ motor vehicle accident. (Reprinted with permission of (59).) osteoporosis declined in the United States from $41 \%$ in 2001 to $21 \%$ in $2011(60)$.

\section{RESPONSE TO THERAPY}

Patients receiving antiosteoporosis therapy should be monitored for compliance, not only with the prescribed drug but also with regard to sufficient calcium and vitamin D. Annual height measurements in this population will not necessarily identify year-to-year changes but can become important over years of assessments. If a patient loses more than $2 \mathrm{~cm}$ in height, vertebral imaging is recommended to evaluate for the occurrence of a new vertebral fracture (29).

BTMs can be used to track not only compliance but also responsiveness (61). These markers include those that reflect bone formation and bone resorption and, thus, bone turnover. In contrast to BMD, which takes several years to change, these dynamic assays can change within 3-6 mo of pharmacotherapy and generally correlate with later changes in BMD (62). For antiresorptive therapies, a reduction of $50 \%$ in BTMs is typically seen in a compliant patient (50). Compliance can be enhanced when patients are aware of their response through demonstrated measures of responsivity, such as BTMs and BMD (63).

Serial DXA testing is also important when monitoring response to osteoporosis therapy. The optimal interval for DXA testing varies and depends on the clinical situation. If one goes strictly by the LSC and expected rate of improvement with most of the therapies available for osteoporosis, an interval of $2 \mathrm{y}$ is reasonable. Yearly monitoring of BMD can be extremely helpful since the drugs can show changes, although these are not always significant within the first year. An observed positive change can also encourage therapeutic compliance (7). Also, a significant reduction in BMD within a year can alert the clinician to an intervening process that is interfering with therapy or a compliance issue. In specific situations such as ongoing glucocorticoid therapy, aromatase inhibitors, or androgen deprivation therapy, yearly measurement of BMD is generally recommended because of the potential for rapid bone loss. For patients receiving long-term antiresorptive treatment with bisphosphonates or denosumab, the ISCD recently recommended considering the use of full-length femur imaging, which uses DXA for the detection of atypical femur fractures, a rare adverse effect of these therapies. On imaging, atypical femur fracture starts as a stress reaction in the subtrochanteric region of the lateral femur (64).

Clinicians may be prompted to investigate possible secondary causes for bone loss if patients are not responding as expected to antiosteoporosis medications. There are many secondary causes of osteoporosis, including rheumatoid arthritis, hyperparathyroidism, untreated longstanding hyperthyroidism, male hypogonadism, premature menopause, malnutrition, multiple myeloma, celiac disease, other malabsorptive disorders, Cushing syndrome, hyperprolactinemia, chronic immobilization, chronic liver disease, osteogenesis imperfecta, type 1 diabetes mellitus, and treatment with certain medications (e.g., antiepileptic, aromatase inhibitor, and glucocorticoid therapies) (5). Obvious secondary causes should have been ruled out before instituting therapy for osteoporosis. This differential diagnosis is a useful reference even if these disorders were not apparent when therapy was instituted.

Drug failure can be considered if a patient has a new fracture on therapy and compliance has been ensured. No drug therapy is perfect; fractures are going to occur despite effective drugs and therapeutic compliance. However, patients understandably often interpret the intercurrent fracture as evidence for drug failure. 
Significantly worsening BMD is a clearer reflection of drug failure, assuming good compliance. If the patient is receiving oral therapy, these situations often call for a switch to parenteral therapy, such as denosumab or zoledronic acid. Another option in patients who have failed antiresorptive therapy by sustaining a fracture is to switch to an anabolic agent, such as teriparatide, abaloparatide, or romosozumab. Limiting factors for the use of anabolic agents for many patients include administration of daily (teriparatide or abaloparatide) or monthly (romosozumab) injection, as well as the cost for treatment.

\section{CONCLUSION}

DXA is a safe and cost-effective method of bone density measurement, both in assessing fracture risk and in monitoring response to therapy. Given the high prevalence of osteoporosis and the morbidity and mortality associated with fragility fractures, BMD measurement remains an important public health intervention.

\section{REFERENCES}

1. Kanis JA, Johnell O, Oden A, et al. Long-term risk of osteoporotic fracture in Malmo. Osteoporos Int. 2000;11:669-674.

2. Panula J, Pihlajamäki H, Mattila VM, et al. Mortality and cause of death in hip fracture patients aged 65 or older: a population-based study. BMC Musculoskelet Disord. 2011;12:105

3. Morrison RS, Chassin MR, Siu AL. The medical consultant's role in caring for patients with hip fracture. Ann Intern Med. 1998;128:1010-1020.

4. Ross PD. Clinical consequences of vertebral fractures. Am J Med. 1997;103(suppl 1): $30 \mathrm{~S}-42 \mathrm{~S}$.

5. Camacho PM, Petak SM, Binkley N, et al. American Association of Clinical Endocrinologists and American College of Endocrinology clinical practice guidelines for the diagnosis and treatment of postmenopausal osteoporosis: 2016. Endocr Pract. 2016;22:1-42.

6. Seeman E. Age- and menopause-related bone loss compromise cortical and trabecular microstructure. J Gerontol A Biol Sci Med Sci. 2013;68:1218-1225.

7. Camacho PM, Petak SM, Binkley N, et al. American Association of Clinical Endocrinologists and American College of Endocrinology clinical practice guidelines for the diagnosis and treatment of postmenopausal osteoporosis: 2016-executive summary. Endocr Pract. 2016;22:1111-1118.

8. Borah B, Gross GJ, Dufresne TE, et al. Three-dimensional microimaging (MRmicroI and microCT), finite element modeling, and rapid prototyping provide unique insights into bone architecture in osteoporosis. Anat Rec. 2001;265:101-110.

9. Njeh CF, Fuerst T, Hans D, Blake GM, Genant HK. Radiation exposure in bone mineral density assessment. Appl Radiat Isot. 1999;50:215-236.

10. World Health Organization. Assessment of fracture risk and its application to screening for postmenopausal osteoporosis: report of a WHO study group. World Health Organ Tech Rep Ser. 1994;843:1-129.

11. Cranney A, Wells G, Willan A, et al. Meta-analyses of therapies for postmenopausal osteoporosis. II. Meta-analysis of alendronate for the treatment of postmenopausal women. Endocr Rev. 2002;23:508-516.

12. El Maghraoui A, Roux C. DXA scanning in clinical practice. QJM. 2008;101: 605-617.

13. Bonnick SL, ed. Bone Densitometry in Clinical Practice: Application and Interpretation. 3rd ed. Humana Press; 2010:1-16.

14. Adult positions. International Society for Clinical Densitometry website. https:// iscd.org/learn/official-positions/adult-positions/. Published May 28, 2019. Accessed March 5, 2021.

15. Huang K, Feng Y, Liu D, Liang W, Li L. Quantification evaluation of ${ }^{99 \mathrm{~m}} \mathrm{Tc}-$ MDP concentration in the lumbar spine with SPECT/CT: compare with bone mineral density. Ann Nucl Med. 2020;34:136-143.

16. Damilakis J, Adams JE, Guglielmi G, Link TM. Radiation exposure in x-raybased imaging techniques used in osteoporosis. Eur Radiol. 2010;20:2707-2714.

17. Boyd S, Müller R. Microimaging. In: Bilezikian JP, Martin TJ, Clemens TL, Rosen CJ, eds. Principles of Bone Biology. 4th ed. Academic Press; 2020:1833-1856.

18. Genant HK, Wu CY, van Kuijk C, Nevitt MC. Vertebral fracture assessment using a semiquantitative technique. J Bone Miner Res. 1993;8:1137-1148.

19. Bone mass measurement: what the numbers mean. National Institutes of Health website. https://www.bones.nih.gov/health-info/bone/bone-health/bone-mass-measure. Updated October 2018. Accessed January 19, 2021.
20. Silva BC, Walker MD, Abraham A, et al. Trabecular bone score is associated with volumetric bone density and microarchitecture as assessed by central QCT and HRpQCT in Chinese American and white women. J Clin Densitom. 2013;16: 554-561.

21. Silva BC, Leslie WD, Resch H, et al. Trabecular bone score: a noninvasive analytical method based upon the DXA image. J Bone Miner Res. 2014;29: $518-530$.

22. Silva BC, Broy SB, Boutroy S, Schousboe JT, Shepherd JA, Leslie WD. Fracture risk prediction by non-BMD DXA measures: the 2015 ISCD official positions part 2: trabecular bone score. J Clin Densitom. 2015;18:309-330.

23. Harvey NC, Gluer CC, Binkley N, et al. Trabecular bone score (TBS) as a new complementary approach for osteoporosis evaluation in clinical practice. Bone. 2015;78:216-224.

24. Liu J, Liu R, Shen X, Zhu G, Li B, Xing M. The genetic duet of BRAF V600E and TERT promoter mutations robustly predicts loss of radioiodine avidity in recurrent papillary thyroid cancer. $J$ Nucl Med. 2020;61:177-182.

25. Kolta S, Briot K, Fechtenbaum J, et al. TBS result is not affected by lumbar spine osteoarthritis. Osteoporos Int. 2014;25:1759-1764.

26. Wildberger L, Boyadzhieva V, Hans D, Stoilov N, Rashkov R, Aubry-Rozier B. Impact of lumbar syndesmophyte on bone health as assessed by bone density (BMD) and bone texture (TBS) in men with axial spondyloarthritis. Joint Bone Spine. 2017;84:463-466.

27. Kim JH, Choi HJ, Ku EJ, et al. Regional body fat depots differently affect bone microarchitecture in postmenopausal Korean women. Osteoporos Int. 2016;27: 1161-1168.

28. Looker AC, Sarafrazi Isfahani N, Fan B, Shepherd JA. Trabecular bone scores and lumbar spine bone mineral density of US adults: comparison of relationships with demographic and body size variables. Osteoporos Int. 2016;27:2467-2475.

29. Cosman F, de Beur SJ, LeBoff MS, et al. Erratum to: clinician's guide to prevention and treatment of osteoporosis. Osteoporos Int. 2015;26:2045-2047.

30. Shuhart CR, Yeap SS, Anderson PA, et al. Executive summary of the 2019 ISCD Position Development Conference on monitoring treatment, DXA cross-calibration and least significant change, spinal cord injury, peri-prosthetic and orthopedic bone health, transgender medicine, and pediatrics. J Clin Densitom. 2019;22: 453-471.

31. Buckley L, Guyatt G, Fink HA, et al. 2017 American College of Rheumatology guideline for the prevention and treatment of glucocorticoid-induced osteoporosis. Arthritis Rheumatol. 2017;69:1521-1537.

32. Stein E, Ebeling P, Shane E. Post-transplantation osteoporosis. Endocrinol Metab Clin North Am. 2007;36:937-963.

33. Leidig-Bruckner G, Hosch S, Dodidou P, et al. Frequency and predictors of osteoporotic fractures after cardiac or liver transplantation: a follow-up study. Lancet. 2001;357:342-347.

34. Shane E, Rivas M, Staron RB, et al. Fracture after cardiac transplantation: a prospective longitudinal study. J Clin Endocrinol Metab. 1996;81:1740-1746.

35. Headley JA, Theriault RL, LeBlanc AD, Vassilopoulou-Sellin R, Hortobagyi GN. Pilot study of bone mineral density in breast cancer patients treated with adjuvant chemotherapy. Cancer Invest. 1998;16:6-11.

36. Ross RW, Small EJ. Osteoporosis in men treated with androgen deprivation therapy for prostate cancer. J Urol. 2002;167:1952-1956.

37. Bhambri R, Naik V, Malhotra N, et al. Changes in bone mineral density following treatment of osteomalacia. J Clin Densitom. 2006;9:120-127.

38. Blake GM. The effect of radiopharmaceutical administration on dual-energy $\mathrm{x}-$ ray absorptiometry scans. J Clin Densitom. 2013;16:257-258.

39. Shepherd JA, Schousboe JT, Broy SB, Engelke K, Leslie WD. Executive summary of the 2015 ISCD Position Development Conference on advanced measures from DXA and QCT: fracture prediction beyond BMD. J Clin Densitom. $2015 ; 18: 274-286$.

40. Ensrud KE, Thompson DE, Cauley JA, et al. Prevalent vertebral deformities predict mortality and hospitalization in older women with low bone mass. Fracture Intervention Trial Research Group. J Am Geriatr Soc. 2000;48:241249.

41. Melton LJ III, Atkinson EJ, Cooper C, O'Fallon WM, Riggs BL. Vertebral fractures predict subsequent fractures. Osteoporos Int. 1999;10:214-221.

42. Siris ES, Adler R, Bilezikian J, et al. The clinical diagnosis of osteoporosis: a position statement from the National Bone Health Alliance Working Group. Osteoporos Int. 2014;25:1439-1443.

43. Reid IR, Horne AM, Mihov B, et al. Fracture prevention with zoledronate in older women with osteopenia. N Engl J Med. 2018;379:2407-2416.

44. Kanis JA, Johansson H, Harvey NC, McCloskey EV. A brief history of FRAX. Arch Osteoporos. 2018;13:118.

45. Briot K, Paternotte S, Kolta S, et al. FRAX: prediction of major osteoporotic fractures in women from the general population: the OPUS study. PLoS One. 2013;8:e83436. 
46. Shoback D, Rosen CJ, Black DM, Cheung AM, Murad MH, Eastell R. Pharmacological management of osteoporosis in postmenopausal women: an Endocrine Society Guideline update. J Clin Endocrinol Metab. 2020;105:587-594.

47. Giangregorio LM, Leslie WD, Lix LM, et al. FRAX underestimates fracture risk in patients with diabetes. J Bone Miner Res. 2012;27:301-308.

48. Whitaker M, Guo J, Kehoe T, Benson G. Bisphosphonates for osteoporosis: where do we go from here? N Engl J Med. 2012;366:2048-2051.

49. Kanis JA, McCloskey EV, Harvey NC, Johansson H, Leslie WD. Intervention thresholds and the diagnosis of osteoporosis. J Bone Miner Res. 2015;30:1747-1753.

50. Stepan JJ, Alenfeld F, Boivin G, Feyen JH, Lakatos P. Mechanisms of action of antiresorptive therapies of postmenopausal osteoporosis. Endocr Regul. 2003;37:225-238.

51. Management of osteoporosis in postmenopausal women: 2010 position statement of The North American Menopause Society. Menopause. 2010;17:25-54.

52. Watts NB, Bilezikian JP, Camacho PM, et al. American Association of Clinical Endocrinologists medical guidelines for clinical practice for the diagnosis and treatment of postmenopausal osteoporosis. Endocr Pract. 2010;16(suppl 3):1-37.

53. Miller PD, Hattersley G, Riis BJ, et al. Effect of abaloparatide vs placebo on new vertebral fractures in postmenopausal women with osteoporosis: a randomized clinical trial. JAMA. 2016;316:722-733.

54. Prevrhal S, Krege JH, Chen P, Genant H, Black DM. Teriparatide vertebral fracture risk reduction determined by quantitative and qualitative radiographic assessment. Curr Med Res Opin. 2009;25:921-928.

55. Canalis E, Giustina A, Bilezikian JP. Mechanisms of anabolic therapies for osteoporosis. N Engl J Med. 2007;357:905-916.
56. Cosman F, Crittenden DB, Adachi JD, et al. Romosozumab treatment in postmenopausal women with osteoporosis. N Engl J Med. 2016;375:1532-1543.

57. Saag KG, Petersen J, Brandi ML, et al. Romosozumab or alendronate for fracture prevention in women with osteoporosis. N Engl J Med. 2017;377: 1417-1427.

58. Langdahl BL, Libanati C, Crittenden DB, et al. Romosozumab (sclerostin monoclonal antibody) versus teriparatide in postmenopausal women with osteoporosis transitioning from oral bisphosphonate therapy: a randomised, open-label, phase 3 trial. Lancet. 2017;390:1585-1594.

59. Adler RA, El-Hajj Fuleihan G, Bauer DC, et al. Managing osteoporosis in patients on long-term bisphosphonate treatment: report of a task force of the American Society for Bone and Mineral Research. J Bone Miner Res. 2016;31:16-35.

60. Solomon DH, Johnston SS, Boytsov NN, McMorrow D, Lane JM, Krohn KD. Osteoporosis medication use after hip fracture in U.S. patients between 2002 and 2011. J Bone Miner Res. 2014;29:1929-1937.

61. Kleerekoper M. Biochemical markers of bone turnover: why theory, research, and clinical practice are still in conflict. Clin Chem. 2001;47:1347-1349.

62. Greenblatt MB, Tsai JN, Wein MN. Bone turnover markers in the diagnosis and monitoring of metabolic bone disease. Clin Chem. 2017;63:464-474.

63. Leslie WD, Morin SN, Martineau P, Bryanton M, Lix LM. Association of bone density monitoring in routine clinical practice with anti-osteoporosis medication use and incident fractures: a matched cohort study. J Bone Miner Res. 2019;34: 1808-1814.

64. Cheung AM, McKenna MJ, van de Laarschot DM, et al. Detection of atypical femur fractures. J Clin Densitom. 2019;22:506-516. 